\title{
Integrating the Quality Requirements into the Riva-Based Business Process Architectures Using the Synthesis of the $i^{*}$ and the NFR Frameworks
}

\author{
Yousra Odeh
}

\begin{abstract}
The Business Process (BP) identification is one of the fundamental and preliminary activities in the Business Process Management (BPM) in order to carry out the design of a Business Process Architecture (BPA). The Riva is a recent BPA modelling approach, which stems from the behavioral business entities. However, a Riva-based BPA lacks the explicit representation of the business Quality Requirements (QRs), which represent the desired quality attributes that constrain the identified BP operations such as security. The Non-Functional Requirements (NFR) Framework is soft Goal-Oriented (GO) approach that systematically elicits NFRs for the development of software systems. The $i *$ framework is another GO approach that tends to understand a business organization. In this paper, a novel approach is proposed in order to integrate the QRs into a Riva BPA using the synthesis of the $i^{*}$ and the NFR frameworks. The work is evaluated using a pilot case study from a health care domain in Jordan. The proposed approach recovered the absence of the QRs in a Riva BPA and enriched its representation. In addition, it optimized the role of the BP identification by addressing both the operational and non-operational aspects. Finally, the generated knowledge from this synthesis is anticipated to assist and benefit the requirement engineers in eliciting the quality of the BP-driven software services.
\end{abstract}

Index Terms-The $i$ * framework, the NFR framework, riva, business process architecture, business process management, quality requirements, quality of service.

\section{INTRODUCTION}

Researchers have put great effort in deriving BPAs from different point of views. By and large, a BPA outlines the key BPs and other associated aspects depending on the notion of the applied BPA method [1]. Although the BPA modelling approaches vary in their directions to obtain a BPA [1], still they do not explicitly address and/or document the desired quality attributes, which constrain the workflow of the identified key BPs (e.g., accuracy). A recent example of this kind of gapis the Riva-based BPAs alignment with goals using the $i^{*}$ framework in order to optimize the BP identification [2]. The resulted Riva BPA model did not address the QRs of the identified goal-based BPs, which many of them run as software services. The origin of this shortcoming sources from the nature of the $i^{*}$ framework [3], that is not an adequate quality-oriented approach for software- based business services. The absence of the QRs in

Manuscript received April 25, 2014; revised July 7, 2014. This work was supported in part by the sponsors of the author's PhD program.

Yousra Odeh is with the Software Engineering Research Group in the University of the West of England, Bristol, BS16 1QY, UK (e-mail: Yousra.Odeh@uwe.ac.uk, Yousra2.Odeh@live.uwe.ac.uk). the Riva-BPA is anticipated to double the effort with regard to the elicitation of the QRs for the BPs' detailed representation. In addition, some of the missing QRs in the Riva-BPA are likely required to derive additional functionalities in the BPs. Hence, the NFR framework [4], which is a structured Soft Goal (SG) or quality-oriented approach for software systems, is capable to conquer the above shortcomings.

In this paper, the authors propose a novel approach that synthesizes the $i *$ and the NFR frameworks in order to bring out a comprehensive Riva-BPA model in both of its functional and non-functional perspectives. Moreover, the novel approach steers the way to optimize the role of the BP identification in the BPM. It also awards bidirectional traceability in order to assist the BP architects in detecting gaps. The work is validated using the pilot case study that is the CD BP in the King Hussein Cancer Care Center in Jordan $[5]$.

The rest of the paper is structured as follows; an essential background is presented in Section II. Section III presents the synthesis of the $i^{*}$ and the NFR frameworks to generate a quality-integrated Riva-BPA (Q-BPA) along with a live evaluation using the $C D$ pilot case study. A rich debate that assesses the work is carried out in Section IV. And last but not least is Section $\mathrm{V}$ that concludes the work

\section{ESSENTIAL BACKGROUND}

This section provides the reader with the required background in order to facilitate the comprehension of the proposed approach. The CD BP pilot case study with a brief but rich description commences this section. Then, the $i^{*}$ framework, the NFR framework and the Riva-BPA with associated example are presented respectively with a short critique afterwards.

\section{A. The Cancer Detection Pilot Case Study}

The cancer detection process, which is a quality-integrated small-scale study, was created to "diagnose patients and determine their cancer type and site" [5]. This pilot study sources from a parent case study in the Kingdom of Jordan, namely the Cancer Care Registration (CCR) process [5]. The $\mathrm{CD}$ process was particularly chosen for this paper in order to assist the authors in completing the promised work, with regard to the quality part, in [2]. Therefore, the brief description of this study is quality-oriented.

Patient, doctor, receptionist, lab and the imaging department are five major roles that collaborate in this process to address the above hard goal. The Non-Functional Requirements (NFRs) were elicited to improve the CD BP 
modlling in [5]. Since the NFRs knowledge sourced from the experts within the CCR, the author deemed the NFR knowledge's sort as a specialized rather than a generalized one. Such specialized NFRs in the CD BP are information domain, information availability, system user satisfaction emapthy, etc. The detailed representation of $\mathrm{CD}$ and its associated NFR catalogues do exist in [5], yet they are not shown in this paper due to the limited space. From those CD NFR catalogues, the reader must pay the attention that the NFR types may be different for a one subject of matter. For instance, the information availability and confidentiality NFRs are required for a one topic that is patient information. In addition, some NFRs are not designable (i.e., not capable to generate quality model) such as empathy. The associated priority of each NFR was not elicited thus this perspective will not be addressed in this paper. Besides, some of the elicited NFRs were not abstracted (e.g., confidentiality). However, the associated quality-integrated BPA, which blueprints the key quality-integrated BPs and their interactions, has not been addressed. In the best case, the quality-integrated BPA was implicitly addressed but not explicitly documented approaches [6].

\section{B. The $i *$ Framework: A Goal-Oriented Approach}

The $i^{*}$ framework is designed to early understand the business process strategies that exist in an organisation in order to elicit the business requirements of the software services, which completely or partially automate many business processes [3]. The framework stands on two types of strategic models that are the Strategic Dependency (SD) and the Strategic Rationale (SR) models [3]. The two models are generated respectively after applying the following steps [3]: 1 - identify the strategic actors in a BP with their associated holding intentions and abilities. Actors are active entities that present either humans (e.g., student) or non-humans (e.g., registration system).2-identify actors, these are dependers, who/which would depend on another actors, those are dependees, in order to obtain their intentions in the mean of dependums (i.e., goal, soft goal, task and resource). In this case, a directed dependency relation of this dependum is created from the depender to the dependee. 3- step one and two are repeated as far as the strategic actors are requiring dependencies.

These two steps generate the SD model that abstractly represents the external dependencies among actors.4- for each dependency relation in the SD model, the strategic rationale relationships within the actors are internally modeled [3]. These relations are the means-end and task decomposition. The first is used when a task requires decomposition into sub elements (i.e., tasks, resources, goals and soft goals). The second is used to link a mean, which is usually a task, to an end in order to represent how an end is fulfilled [3]. 5- step 4 generates the SR model and is repeated as far as the internal rationale representations are required. In reality, the aforementioned steps are iterated and carried out in parallel [3]. However, they are ordered to facilitate the comprehension of the $i^{*}$ framework.

The author advocates her selection of the $i *$ framework in this work due to a set of relevant reasons. Firstly, the $i^{*}$ framework has initially demonstrated its capability in deriving and re-engineering a BPA and associated BPs models in [2], [7]. However, it has not yet integrated QRs into the corresponding BPA model. Secondly, the author founds the $i^{*}$ framework is rich of early quality business-oriented concepts to harmonize with a SGO approach in order to extend a BPA with traceability in both directions. Last but not least, the $i *$ framework is odd among other GO approaches due to its systematic execution and reasonable notion in understanding an organization using strategic dependency networks

\section{The NFR Framework: A Soft Goal-Oriented Approach}

The NFR framework is a SGO approach that aims at linking soft goals (i.e., QRs) into software systems or their models using SIGs with full bidirectional traceability. This framework is the most recognized and acknowledged quality-based approach in software engineering and development [4]. The SIG graph for a particular NFR is generated after carrying out the following steps [4]: 1-obtain the required knowledge with regard to the system's domain, its functional requirements and NFRs with their eligible techniques. 2-identify the top or major NFRs of the system, namely the main soft goals. Each soft goal consists of a NFR (e.g., security, user-friendliness) and an associated subject of matter parameter, namely target (e.g., flight booking). 3-refine the SG downwards systematically into sub soft goals or sub goals to some extent, which is determined by the requirement engineers and developers. The offspring soft goals contribute upwards using AND or OR interdependencies to meet the parent. This elaboration is likely processed in a top-down fashion. The elaboration and contribution are required to eliminate NFR meaning's ambiguity. 4-determine the priority soft goals that must be successfully addressed in the first place.5- derive the operationalizations, which are data, constraints and operations, that actually fulfil the associated NFR parent. 6 deal with both positive and negative interdependencies that are either explicit or implicit between the soft goals. The aforementioned steps are not sequential. Besides, each step in the framework iterates as far as required [4].

The NFR framework is characterized as a soft goal independent framework, which adapts with various NFR types, both general and specialized, to produce their SIGs [4]. It is a systematic method and rich of the quality-based concepts, which implicitly embody the Quality of Service (QoS). Consequently, those features have facilitated the NFRs elicitation and maintaining their SIGs in catalogues for reuse. However, the NFRs' business, beyond, rationales were not highlighted. In addition, the engagement of this framework in the BPM, and particularly in a Riva-BPA, to develop a business-based software services is very few [5]. Therefore, an elegant synthesis with the $i *$ framework, which aims to strategically understand the organization's structure, would assist in addressing the two aforementioned concerns.

\section{The Riva-Based Business Process Architecture}

By and large, the BPA blueprints a collection of key BPs and their interactions of an organization, which it could be a process or even a group of individuals. The BPA modelling approaches are classified into five categories: 1) goal-based, 2) action-based, 3) object-based, 4) reference model-based and (v) function-based approaches [1]. For example, the Riva-BPA method is classified as an object-based BPA 
modelling approach [1]. It blueprints the dynamic BPs with their relationships, which are continuously changing to meet business needs. The identified BPs originates from hard business terms, namely the Essential Business Entities (EBEs).

The Riva method outputs the BPA model after carrying out the following steps [8]: 1- elicit EBEs, which are the subject of matter that characterize the business an organization is in, through brainstorming process and reserve them in a list, namely the EBE list. The list consists of abstract EBEs (e.g., account) and physical ones (e.g., phone). 2- review the EBEs' list and determine EBEs that hold a life time required by the organization. We call the filtered EBEs with their associated lifetime, Unit of Works (UoWs). Put the resulted UoWs in the UoWs list. The reader must recognize that the UoWs list is subset from the EBEs list. 4- establish the UoW diagram using the corresponding UoWs list. A UoW is interrelated with one another through dynamic relationship, which is the "generates" relation. This relation is directed from the source UoW to the destination UoW and labeled with one of the cardinalities, 1:1 or 1:many. 5- carry out some principles in order to create the $1^{\text {st }}$ cut BPA from the UoW diagram. Each UoW in the diagram creates a corresponding $\mathrm{BP}$ in the $1^{\text {st }}$ cut BPA, namely Case Process (CP). Besides, the Case Management Process (CMP) is BP that manages the generated instances of a particular CP. Thus, the UoW diagram predicts the optimal BPA model [2], [8]. 6-finally, the Riva BPA model is obtained after reducing the $1^{\text {st }}$ cut to the $2^{\text {nd }}$ cut BPA by following a set of heuristics in [8]. Although the Riva method models the abstraction of BPs, it also designs the detailed representation of each BP in the BPA using role-oriented BP modelling (e.g. BPMN and RAD) [6], [8], [9].

From the steps above, the author adopted the Riva BPA method due its easiness in establishing a BPA models. It is systematic, easy to understand, rich of relevant business-oriented concepts, and highly adaptable to the business changes. As a recent example, the author has reengineered the Riva-BPA using the $i *$ framework with full traceability [2]. However, the Riva method lacks the QRs representation which exhibits how the QRs or soft goals constraint the operation of a BP.

\section{1) The cancer detection riva-based $B P A$}

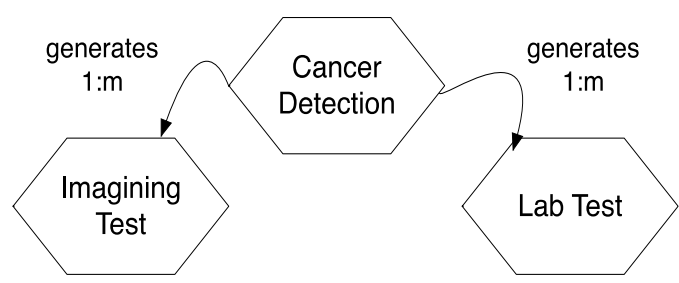

Fig. 1. The UoW diagram of the cancer detection pilot study.

This sub section applies the steps above in order to generate the CD Riva-based BPA. Since the UoW was found useful to predict the optimal BPA model, the author decided to employ the UoW diagram, in this paper, instead of proceeding with the late detailed Riva-BPA design steps. Besides, the CD case study is employed as a pilot. Therefore, the CD UoW diagram was designed using 3 UoWs from 24 EBEs [6], [2]. The information availability is a desired quality attribute that constrains the $\mathrm{CD}$ process execution as it has been presented in section II A [5]. Fig. 1 depicts the CD UoW diagram that lacks the visualization of the information availability QR.

III. GENERATING THE RIVA-BASED QUALITY-LINKED BPA FROM THE SYNTHESIS OF THE $I^{*}$ AND THE NFR FRAMEWORKS WITH THE REFERENCE TO THE CANCER DETECTION EXAMPLE

This section aims to exhibit the synthesis of two GO approaches, that are the $i *$ and the NFR frameworks, in order to generate the Riva-based Q-BPA. In order to fulfil this aim, two-core stages process must be carried out as shown in Fig. 2 . In addition, the process is presented and supported with the actual models using the CD pilot case study. However, all models are partially designed and illustrated due to the limited space of this paper and to reduce the complexity of unwanted details.

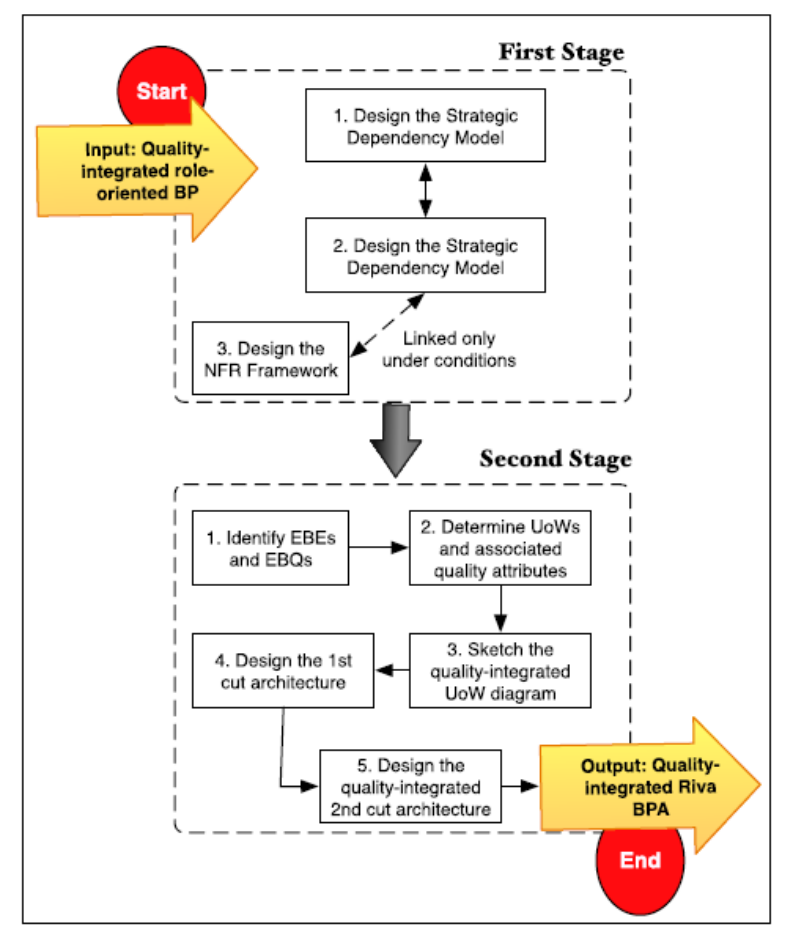

Fig. 2. Synthesizing the $i^{*}$ framework and the NFR framework along with a refinement of the original Riva BPA modeling method.

The process inputs a quality-integrated role-oriented BP (e.g., CD BP) in order to generate the GO models, which assist to output a Q-BPA. In Fig. 2, the top dotted rectangle refers to the first core stage that is designing the synthesis, if required, of both GO approaches. Three activities constitute this first stage: (1) design the SD model, (2) design the SR model and (3) design the NFR framework if required. In this stage, the NFR framework is only designed for SGs (i.e., QRs) within the boundary of the $i *$ framework actors that many of their roles are automated in the form of software services. Otherwise, there is no need to design the NFR framework and the business analyst have to only deal with the $i^{*}$ framework models. Hence, this explains why the author denoted the synthesis by a bidirectional dotted arrow in Fig. 2 . The three activities overlap and iterate as shown in Fig. 2. The bottom dotted rectangle is the second core stage as shown in Fig. 2. This stage refines the original Riva method [8] in order to result the Riva Q-BPA. Five activities constitute the second stage: 1) identify EBEs and associated EBQs, () determine 
the UoWs and their associated 3) sketch the quality-integrated UoW diagram, 4) design the $1^{\text {st }}$ cut architecture and 5) design the $2^{\text {nd }}$ cut architecture, namely the Riva Q-BPA.

\section{A. The First Stage: Synthesizing the Goal-Oriented Models}

This stage aims at designing the GO models, from the quality-integrated and role-oriented BPs, in order to extend the Riva-BPA with the SG rationales through carrying out the three activities, as they are illustrated in Fig. 2. In this stage, it is compulsory to design the both strategic models of the $i^{*}$ framework. However, the NFR framework is only designed under particular linking conditions, which are presented in the third sub section of this stage.

\section{1) Designing the strategic dependency model}

The SD model is the first goal-oriented blueprint that emerges in the proposed approach. This sub-phase applies the SD modelling steps that were presented in Section II [3]. For each business process there is a corresponding SD model and an associated SR model that elaborates the former [2], [3]. In the SD model, hard goal and soft goal dependencies are only designed where the rest will be derived later during the modelling of the corresponding SR. This is because of the notion of abstraction of goals and soft goals that are higher than the rest business-oriented terms, which are candidate operationalizations in the corresponding SR model.

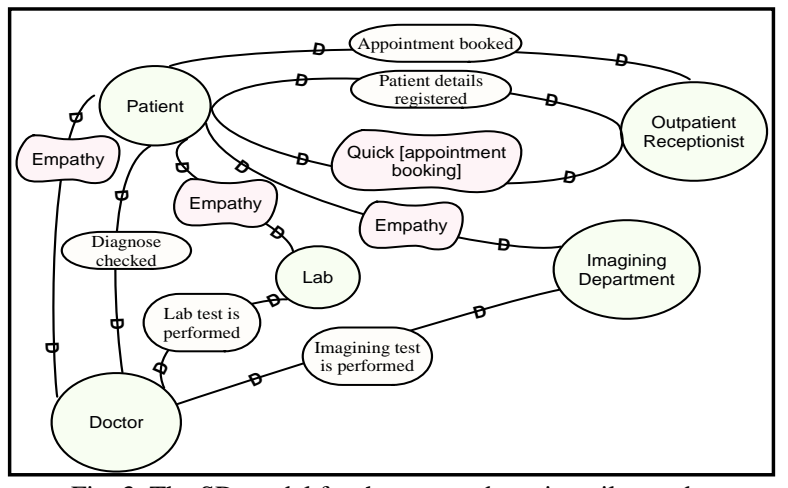

Fig. 3. The SD model for the cancer detection pilot study.

Fig. 3 depicts a partial CD SD model with its goal and soft goal dependencies. In this Fig., the patient depends on the receptionist for the quick appointment booking, which requires a soft goal dependency relation. The patient depends on doctor, lab and imagining test to provide him/her with empathy as a soft goal dependum in a soft goal dependency relation. Moreover, the doctor depends on the investigation team for investigations to be performed. Hence, this is designed as a goal dependency relation as shown in Fig. 3.

\section{2) Designing the strategic rationale model}

This activity aims to elaborate the above corresponding SD model. The internal rationale modelling steps are exactly followed, as presented in Section II [3]. However, few conditional linking cases change the internal structure of an actor as shown in Fig. 4. The first linking case when a soft goal dependum is addressed via its corresponding SIG within the dependee's boundary. The second linking case is when the SIG is sub of a task. In another words, this case means that the main SG is constraining the task parent. The third and the fourth linking cases state that if an operationalization within a SIG matches with a task or resources then, the analyst must give the priority for the NFR framework design in order to exhibit the linkage by reserving the operationalization as shown in the cases c and d in Fig. 4.

A partial CD SR model is illustrated in Fig. 5. In Fig. 5, the four goal dependencies and three soft goal dependencies are addressed within the actor boundaries doctor, receptionist and lab through carrying out the steps presented in Section II B.

\section{3) Designing the NFR framework for the soft goals within the SR model}

This activity aims to design the NFR framework in order to operationalise the desired synthesis. It is described as a conditional activity. In fact, the NFR framework is married to the $i *$ framework if any of the four conditions is valid. In other words, the reader must not apply this phase if the linking case is not shown up. Fig. 2 depicts this conditional case using the dotted arrow in the top rectangle.

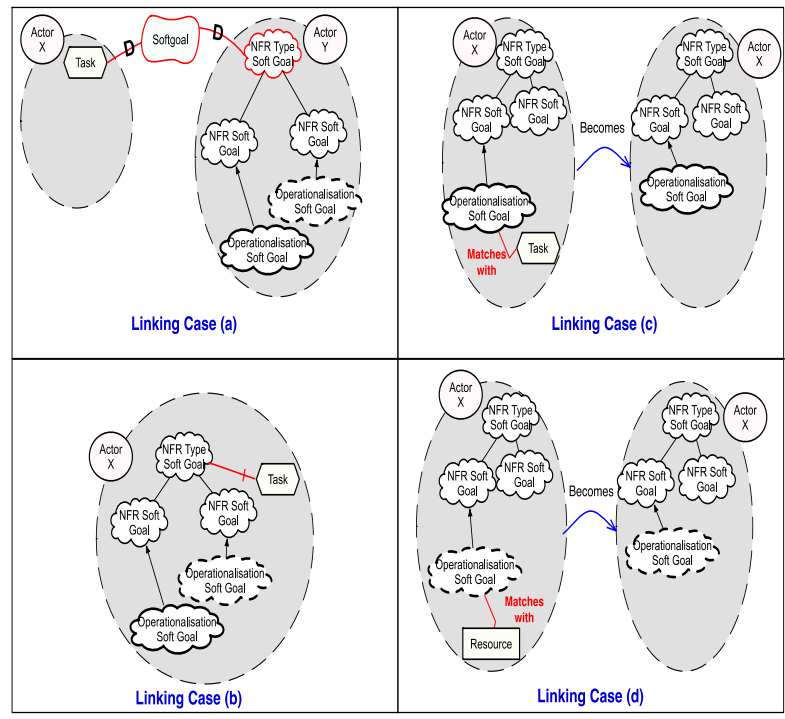

Fig. 4. The four cases that link a SIG for a NFR within a SR model.

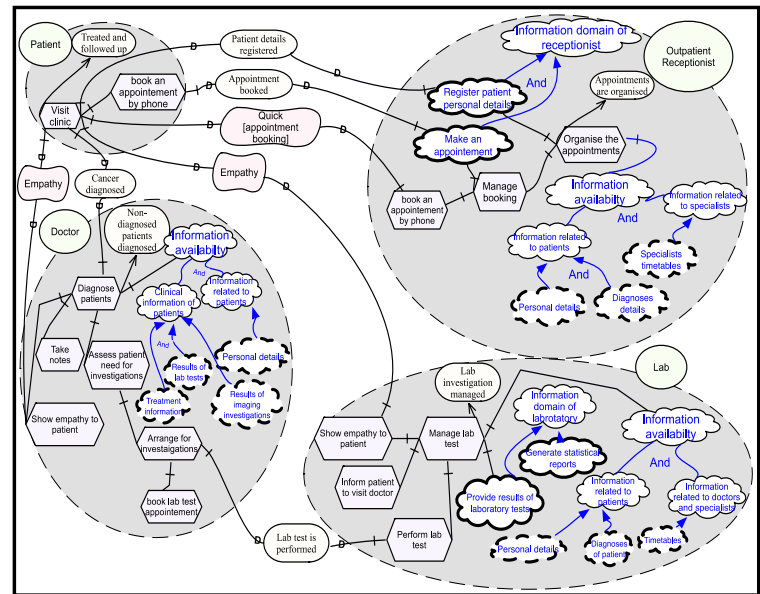

Fig. 5. Partial CD SR model of the $i^{*}$ framework with the synthesis of the SIG of the NFR framework.

The first two steps, which were presented in Section II, of the NFR framework were informally carried out through texts. At present, in the previous phases, the $i^{*}$ framework does semi formally execute them using its rich strategic models. The remained steps, with regard to the dealing with the decompositions and interdependencies, of the NFR framework are performed as they are in [4]. However, at the 
current stage of this research, the work does not address the priority perspective. Thus, the authors extend the SR modeling relations to handle this linking guided by the cases in Fig. 4.

In Fig. 5, the information availability SIGs linked within the doctor, receptionist and lab boundaries using the second, third and fourth linking case presented in Fig. 4.

Finally, the reader must be aware/alerted that the NFR framework does not derive the Riva-based BPA development, yet it explicitly and systematically addresses and documents the missing quality module in the Riva BPA.

\section{B. The Second Stage: Designing the Riva-Based Quality-Integrated BPA Using Stage 1}

This stage utilizes the rich strategic knowledge that was generated in stage one in order to produce the Riva-Q-BPA model, as shown in Fig. 2. Since the UoW diagram was found useful to predict the optimal BPA model, the author decided to employ the UoW diagram, in this paper, instead of proceeding with the late detailed Riva-BPA design steps. In addition, the CD case study is employed as a pilot, which supports the author's decision with regard to the UoWs diagram predictability feature. Therefore, the fourth and the fifth activity in Fig. 2 are not addressed at this stage of work.

\section{1) Identifying the EBEs and their associated quality entities using the goal-oriented strategic models and knowledge}

This activity aims to identify the EBEs that form the source of the promised Riva-Q-BPA, with disregard if they are derived or detected as in [2]. The EBEs identification guidelines are followed as they are in [8].

The author proposes a new quality concept in the Riva method as a sibling for the EBEs, namely the Essential Business Quality (EBQ). An EBQ represents the main desired QR attribute or constraint on all or parts of the EBEs that both stem from the understanding of the business an organization is in. It is necessary to differentiate between a main QR and the mechanism that address the QR. In particular, the former is called EBQ where the latter is called Designed Business Quality (DBQ). For example, confidentiality is an EBQ where the encryption is a DBQ as a alternative mechanism for addressing the EBQ.

\section{2) Determining the UoWs and their associated quality attributes}

This activity aims to filter the UoWs from the EBEs list and identify their associated SGs or QRs. This generates a new term to the Riva method, namely Quality on the UoW (Q-UoW). The author defines this new term, which is the Q-UoWs, as the desired business quality requirement or attribute that holds a lifetime the organization is interested in the corresponding UoW. The QR or SGs are detected from the generated knowledge in the first stage Thus, the UoWs list is now extended and it lists the UoWs and associated Q-UoWs that constrain their execution within the lifetime. For example, the CD study contains 5UoWsinstead of 3 as was shown in Fig. 1. The information availability and empathy SGs constrain the five UoWs. Hence, they are Q-UoWsnow.

\section{3) Sketching the quality-integrated UoW diagram}

This activity aims to explicitly document the QR in the Riva-BPA. The UoWs and associated Q-UoWs list facilitates the addressing of aim above. Although the UoWs were interrelated to each other's via dynamic operational relationships, still they are not interrelated using non-operational ones. Therefore, in this activity the author extends the UoW diagram by adding three new notations that are Q-UoW, which is denoted in the UoW diagram by a double lined gray hexagon or dotted hexagon, and the dynamic quality constraints relationship, which is denoted by a curvy dotted line without cardinality. The Q-UoWis either associated with quality model (i.e., represented using the double hexagon) reference or not (i.e., represented using the dotted hexagon). In particular, the former is considered as an elaborative QR and thereby designed using quality modeling method such as the NFR framework (e.g., information availability). Furthermore, this kind of Q-UoWs must explicitly associate the quality reference model between brackets. The latter cannot derive its quality model due to tis abstraction notion (e.g., empathy).

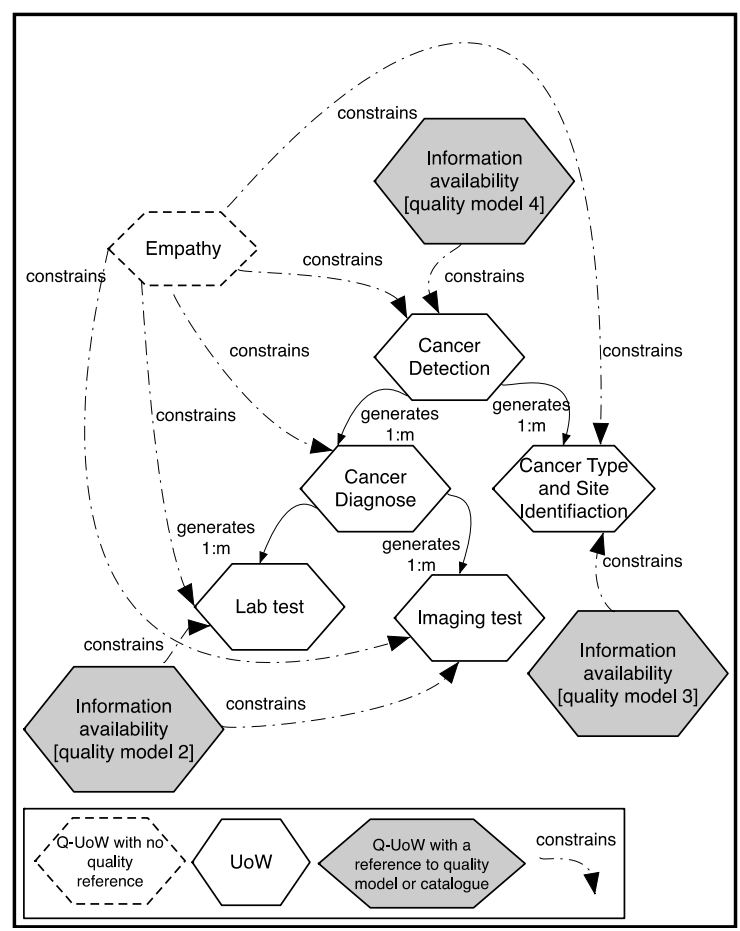

Fig. 6. The quality-integrated UoW diagram from the cancer detection pilot study.

The quality integrated UoW diagram of the CD process is shown in Fig. 6. The Q-UoWsare the information availability and empathy that constrain the rest essential goal-based UoWs.

\section{DISCUSSION}

This section discusses the already presented novel approach, which has resulted an extended and enriched Riva-based BPA using the GO and the SGO approaches. In addition, the section involves a brief debate, yet it is rich by highlighting the merits, findings' implications and limitations. The author presents them as lessons that have been learned during the work.

The first lesson teaches the reader that s/he must pay attention for the elicitation carried out for the QRs. It did not commence during in the BPA development, yet the author utilized the QRs that live in the GO models. 
Second, the harmony of the whole integration with regard to the two goal-oriented approaches and the Riva method has enhanced the quality requirements elicitation activity in the requirements engineering process. Thus, the quality requirement engineers with the business architects are now able to systematically elicit the NFRs for software systems.

This is anticipated to complement the work in [2], [10]. In particular, the work has optimized the Riva BPA model by documenting its integration with business QRs, which embody the QoS requirements for candidate software services [6].

Third, the heterogeneous of GO approaches has enriched the Riva BPA model in terms of addressing the operational and non-operational perspectives in this business-oriented context. Hence, the new representation of Riva BPA is an ideal candidate model with the associated BPs to assist the business experts and software experts in establishing a business-based software requirements specification. Besides, the involved stakeholders, who depend on the specification deliverable, are anticipated to benefit from this specification in carrying out their development activities afterwards (e.g., software design and testing). Accordingly, the approach has enriched the software development process.

Fourth, at present, the BP identification activity carries out the identification of key BPs, their QRs and their relations. The BPM development process is now as well enriched and optimized through eliciting the business quality requirements during its early stages using GO models [11].

Fifth, the synthesis has enhanced the first two steps execution in the NFR framework. The authors have shown how they were semi-formally addressed using the $i^{*}$ framework models. This shift is anticipated to bring out a common communication language between stakeholders. Moreover, the visualized models are likely better for understanding than texts.

Sixth, the synthesis of the two GO approaches, that are the $i^{*}$ and the NFR frameworks, has established a rich resource of domain knowledge for the business/IT organizations. The resource was found valuable for use for the BPA and the associated BPs development and management. Moreover, it is anticipated to be valuable for the re-use for their reengineering conditions [2]. With regard to the business organization, this domain knowledge reserves its understanding. With regard to the IT part in the organization, the knowledge repository embodies the software system specification that is anticipated to automate many of the organizational BPs.

This work is limited for individual organizations that are not depending on another in achieving their goals. In the current stage, the approach is carried out manually in eliciting the business quality requirements that constraint the fulfillment of organizational hard goals. In addition, the approach does not mitigate any emergent risks. They are likely to emerge within the goal-oriented approaches if an actor failed to deliver the required dependum and this is anticipated to leave a shortcoming or lack in the Riva BPA's development.

\section{CONCLUSION}

The paper has presented the integration of quality requirements into the Riva-BPA model, regardless the pre-existence of the Riva model, using the synthesis of two GO approaches, which are the $i^{*}$ and the NFR frameworks. This work has filled the missing required module of the Riva-BPA, which is the desired business quality attributes. As overall, the work formed an attempt towards delivering a comprehensive Riva-BPA model with regard to its functional and non-functional perspectives. The cancer detection pilot case study validated this work.

The work has highlighted on the adaptability of the Riva method through its welcoming for new and foreign business-oriented concepts. Moreover, the Riva BPA model bridged the gap between the hard \& soft GO models and the BPA models\& associated BPs. This result is anticipated to complement the work of bridging the gap between business processes and software systems. The bridged gap has alerted the requirements engineers and $\mathrm{BP}$ architects to elicit the QRs from the GO approaches rather than postponing it to the stage of the detailed design of BPs. Therefore, this is anticipated to result a better cost estimation with regard to the quality requirements integration and development cost. By and large, this paper attempts to improve the software development process and the BPM process and particularly its BP identification phase.

In the near future, the author plans to automate the QRs elicitation process using OWL-DL. This will reserve the already elicited QRs in one repository and reuse them in order to enhance the communication between the stakeholders. In addition, the work will be integrated into the BPAOntoSOA framework and enterprise information architectures. Risks, associated mitigation mechanisms and business/IT requirements priority are anticipated to occupy a good space of the future work. In addition, reengineering a Riva-based BPAs, with regard to both of its operational and non-operational modules, using the $i^{*}$ framework will be an attempt to bring a rigorous alignment methodology, which will be employed in a dynamic business/IT environment. In addition, delivering a comprehensive QRs classification for the BPA models will take another part of the future work. Moreover, the author plans to utilize the proposed synthesis of the GO approaches in order to measure the cost estimation of the Riva-Q-BPA model prior heading to its development Finally, the author looks forward transferring this work to the cloud paradigm as a service for a wider application.

\section{REFERENCES}

[1] R. Dijkman, I. Vanderfeesten, and H. Reijers, "The road to a business process architecture: An overview of approaches and their use," Technical Technische Universiteit Eindhoven Report, Beta Working Papers Series, WP-350, 2011.

[2] Y. Odeh, M. Odeh, and S. Green, "Aligning riva-based business process architectures with business goals using the $i^{*}$ framework," in Proc. 3rd International Conf. Business Intelligence and Technology, IARIA, 2013, pp. 16-20.

[3] E. Yu, P. Giorgini, N. Maiden, and J. Mylopoulos, Social Modeling for Requirements Engineering, 1st ed., London, UK: MIT Press, 2010, ch. 2-3.

[4] L. Chung, B. Nixon, and J. Mylopoulos, Non-Functional Requirements in Software Engineering, London, UK: Kluwer Academic Publishers, 2000 .

[5] F. Abu Rub, M. Odeh, and I. Beeson, "Modelling non-functional requirements of business processes," Journal of Information and Software Technology, vol. 49, pp. 1073-1172, 2007.

[6] R. Yousef, M. Odeh, D. Coward, and A. Sharieh, "BPAOntoSOA: Generic framework to derive software service oriented models from 
business process architectures," in Proc. 2nd International Conf Applications of Digital Information and Web Technologies (ICADIWT'09), 2009, pp. 50-55.

[7] G. Grau, X. Franch, and N. A. M Maiden, "PRiM: An $i *$-based proces reengineering method for information systems specification," Information and Software Technology Journal, vol. 50, issue 1-2, pp. 76-100, 2007.

[8] S. Green and M. Ould, "The primacy of process architecture," in Proc. CAiSE Workshops, 2004, vol. 2, pp. 154-159.

[9] S. White and D. Miers, BPMN Modeling and Reference Guide, 1st ed., Florida, USA: Future Strategies Inc., 2008.

[10] M. Odeh and R. Kamm, "Bridging the gap between business process models and system," Information and Software Technology: Special Edition on Modeling Organizational Processes, vol. 45, no. 15, pp. 1053-1060, Dec. 2003

[11] M. Dumas, M. L. Rosa, J. Mendling, and H. A. Reijers, Fundamentals of Business Process Management, 1st ed., London, U.L: Springer, 2013.

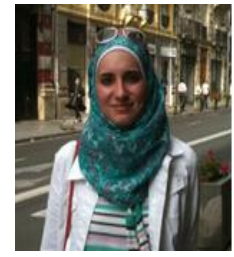

Y. Odeh was born in January 1989 in the Kingdom of Saudi Arabia and holds a BSc degree in the computer science from the University of Jordan in the Kingdom of Jordan.

She is currently at the end of her $\mathrm{PhD}$ study program in the University of the West of England (UWE), Bristol, United Kingdom. Her $\mathrm{PhD}$ is interested in the software engineering field and particularly in the requirements engineering, goal-oriented modeling approaches, business process architecture and modeling, Business/IT alignment, ontologies and software service oriented applications. During her $\mathrm{PhD}$ journey, she published few conference papers. She is interested to join the academic field in the near future and share her experience and knowledge in the software engineering. Yousra Odeh is a member in the BUSTECH committee. 